\title{
A dedicated polarimeter for the MuSiCoS échelle spectrograph
}

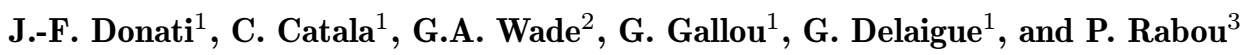 \\ 1 Laboratoire d'Astrophysique, Observatoire Midi-Pyrénées, F-31400 Toulouse, France \\ 2 Department of Physics and Astronomy, The University of Western Ontario, London Ontario, Canada N6A 3K7 \\ 3 Laboratoire d'Astrophysique, Observatoire de Grenoble, 414 rue de la Piscine, BP. 53, F-38041 Grenoble Cedex 9, France
}

Received May 18; accepted July 16, 1998

\begin{abstract}
We present in this paper the technical characteristics of a new polarimetric unit dedicated to the $\mathrm{MuSiCoS}$ échelle spectrograph. The first test runs indicate that our instrument is very good at measuring polarisation or depolarisation structures in line profiles. It is therefore one of the very few facilities worldwide for studying magnetic topologies of active and chemically peculiar stars through rotational modulation of linearly and circularly polarised Zeeman signatures in line profiles. It is also a very interesting tool for investigating geometries of non-axisymmetric circumstellar environments through depolarisation of spectral lines formed within the scattering envelope.
\end{abstract}

Key words: stars: magnetic fields - stars: circumstellar matter - line: profiles - polarization — instrumentation: polarimeters

\section{Introduction}

The MuSiCoS (Multi-Site Continuous Spectroscopy) programme (Catala et al. 1993) is an international collaboration aimed at monitoring the spectral variability of stellar atmospheres on both short and intermediate timescales. This project, gathering about 100 astronomers from 15 countries on five continents, has started to set up a worldwide network of $2 \mathrm{~m}$ class telescopes equipped with identical échelle spectrographs. MuSiCoS began in 1990 with the development of a prototype fibre-fed cross-dispersed spectrograph, designed to yield the whole visible spectrum of a star (from 390 to $870 \mathrm{~nm}$ ) in two exposures, with a mean resolving power of about 35000 (Baudrand \& Böhm 1992). This transportable spectrograph has been involved in three MuSiCoS campaigns to date (in 1992, 1994 and 1996) during which it was installed in key sites (Hawaii and South Africa) where such instrumentation

Send offprint requests to: J.-F. Donati was not available. Between these campaigns, the MuSiCoS spectrograph is installed at the $2 \mathrm{~m}$ Télescope Bernard Lyot (TBL) at Pic du Midi and available to the entire astronomical community. The MuSiCoS spectrograph has been duplicated twice already and the two copies are currently installed on the $2.5 \mathrm{~m}$ Isaac Newton Telescope (La Palma) and the $1.9 \mathrm{~m}$ of the South African Astronomical Observatory (Sutherland).

The present paper focuses on the second logical step of the MuSiCoS instrumentation plan: the development of a dedicated Cassegrain polarimetric unit for the MuSiCoS échelle spectrograph. Numerous scientific programmes can indeed benefit from such an instrument and take advantage of the multiplex gain in effective $\mathrm{S} / \mathrm{N}$ associated with the large spectral domain one can collect in a single exposure. Using "Least-Squares Deconvolution" (LSD, a new cross-correlation technique devised by Donati et al. 1997), one can for instance try to detect circularly and linearly polarised Zeeman signatures in line profiles of both active solar-type and chemically peculiar stars, in order to obtain information about their surface magnetic field topologies (e.g. Donati \& Cameron 1997). One can also study stellar flares and the associated atmospheric particle beam bombardments, by looking for time dependent linear polarisation in several Balmer lines simultaneously (e.g. Saar et al. 1994).

A spectropolarimetric device can also be useful to measure the chromatic variation of continuum linear and circular polarisation from diffusing circumstellar envelopes and/or discs and therefore estimate the size, shape and chemical composition of the diffusing grains, as well as the large-scale geometry and structure of the scattering environment (e.g. Chrysostomou et al. 1997). Similarly, studying how different emission lines (and in particular forbidden lines) are depolarised with respect to the surrounding continuum can provide strong observational constraint on where these lines form with respect to the scattering medium. 
This new polarimeter was designed and constructed at Observatoire Midi-Pyrénées in 1996, with CNRS/INSU (Centre National de la Recherche Scientifique, Institut National des Sciences de l'Univers), MENESR (Ministère de l'Éducation Nationale, de l'Enseignement Supérieur et de la Recherche), Région Midi-Pyrénées and OMP (Observatoire Midi-Pyrénées) funds. In this paper, we first present the technical characteristics of this new instrument (see Sect. 2), then describe how it performs in the particular case of the scientific programmes listed above (Sect. 3) and conclude on its potential involvement in future MuSiCoS campaigns.

\section{Description of the instrument}

The new MuSiCoS polarimeter is inspired by the prototype instrument built by Semel et al. (1993). It is designed to be mounted at the Cassegrain focus of a $2 \mathrm{~m}$ telescope through an interface module which contains all of the usual spectroscopic calibration (halogen and thorium/argon lamps) and guiding facilities.

The optical layout of the instrument is shown in Fig. 1. A detailed description of the different optical components, as well as of the mechanical and electronic design is given in the following paragraphs. All optical surfaces are coated with multilayer antireflection films ensuring a reflectance everywhere lower than 1.5\% (and lower than 1\% in average) throughout the whole spectral domain (390 to $870 \mathrm{~nm})$.

\subsection{Entrance aperture}

The stellar light is collected through a $2^{\prime \prime}$ circular aperture placed at the Cassegrain focus, which is followed by a short focal length doublet that converts the beam to a speed of $\mathrm{f} / 25$. In the particular case of the TBL (which already has an $\mathrm{f} / 25$ Cassegrain beam), this aperture is $500 \mu \mathrm{m}$ in diameter and no attendant doublet is used. For an hypothetic f/ 8 Cassegrain beam for instance, a $160 \mu \mathrm{m}$ aperture should be used, and a Melles Griot achromatic doublet LAO001 (with $10 \mathrm{~mm}$ focal length) located $4.44 \mathrm{~mm}$ behind would achieve the requested beam speed conversion.

\subsection{Polarisers}

Two sheet polarisers (installed in a rotatable wheel with three positions) can be inserted into the beam (slots 1 and 3 correspond to the circular and linear polariser respectively, while slot 2 is left empty). The linear polariser is oriented at a fixed azimuth in the polarimeter frame, defining a reference azimuth throughout the whole instrument.

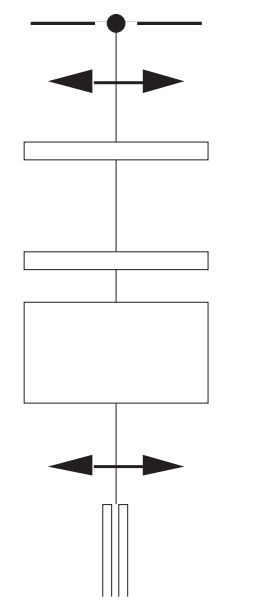

Cassegrain focus, entrance aperture F/25 conversion

Linear or circular polariser

Half-wave or quarter-wave plate

Beamsplitter

Focal reducer $\mathrm{f} / 25-\mathrm{f} / 2.5$

\section{Optical fibres}

Fig. 1. Optical layout of the MuSiCoS polarimeter

\subsection{Wave plates}

Two wave plates (installed in a second rotatable wheel with three positions) can also be inserted into the beam (slots 1 and 3 correspond to the quarter-wave plate and half-wave plate respectively, while slot 2 is left empty). The half-wave plate can be rotated to angles of $0^{\circ}, 22.5^{\circ}$, $45^{\circ}$ and $67.5^{\circ}$ with respect to the reference azimuth (see Sect. 2.2), while the quarter-wave plate can be oriented at angles of $-45^{\circ}, 0^{\circ}$ and $45^{\circ}$. Note that the beam aperture (f/25) is sufficiently small that the associated error in wave plate retardance can be neglected.

The superachromatic wave plates built by the German company Bernard Halle Nachfl. (made of three pairs of quartz and $\mathrm{MgF}_{2}$ plates cemented between parallel plates of fused silica, following the design of Serkowski 1974) are the only commercial crystalline retarders with close to nominal retardance and fast axis direction (within about $1 \%$ and $4 \%$ for the half-wave plate and quarter-wave plate respectively) throughout the whole spectral domain of interest (390 to $870 \mathrm{~nm}$ ). However, we discovered that these wave plates introduce large ripples both in polarisation and intensity spectra (with respective peak-to-peak amplitudes of about $1 \%$ and $2 \%$ respectively), most likely due to interference within the multiple cement layers of the retarders. This is very likely a general problem of all Halle superachromatic retarders (rather than a specific defect of ours as we first thought), since all similar plates that we know of (used in the polarimetric modules at the AngloAustralian Telescope and William Herschell Telescope) also generate such ripple (e.g. Harries \& Howarth 1996). The analytical modeling proposed by Harries \& Howarth (1996) to correct the spectropolarimetric data from this ripple, although successful at a spectral resolution of 5000 , turns out to be insufficient at spectral resolution of 35000 given the very complex shape and multiperiodic behaviour of the fringe pattern (see Fig. 2). Moreover, this ripple is found to vary significantly in position and shape on a timescale of only a few hours (probably with dome 


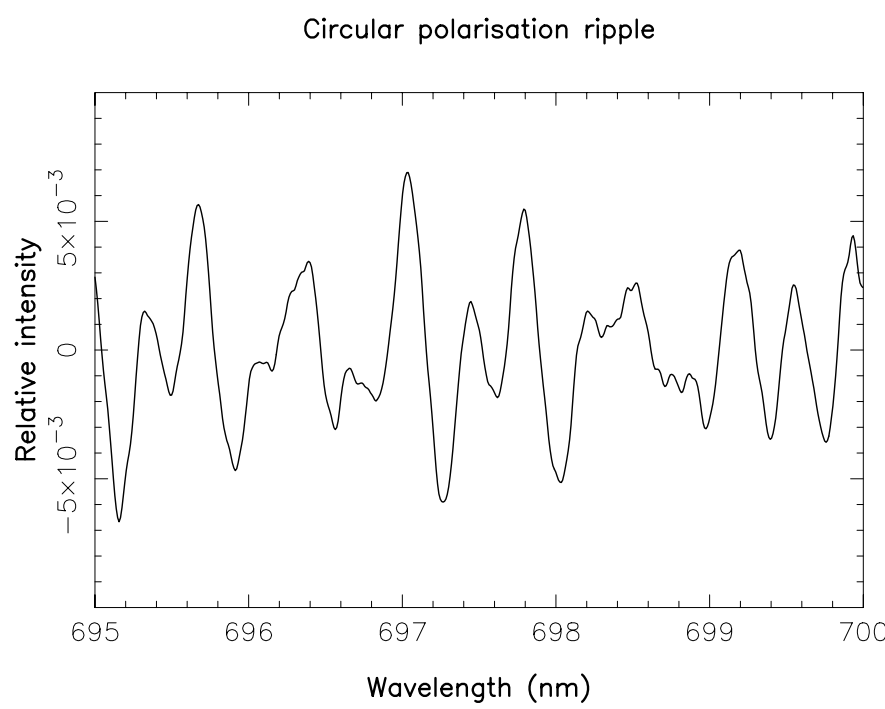

Fig. 2. Polarisation ripple in Stokes $V$ spectra generated by the Halle superachromatic quarter-wave plate

temperature); removing this effect using spectra of a bright unpolarised standard is therefore not a viable solution either.

We therefore decided (as a temporary solution, hoping that the Halle wave plate problem is correctable) to use an achromatic retarder built by the French company Optique Jean Fichou (made of one quartz and one $\mathrm{MgF}_{2}$ crystal plate cemented together with axes at right angles) as a quarter-wave plate, whose deviation from nominal retardance is lower than about $3 \%$ in a more restricted spectral domain (i.e. 400 to $700 \mathrm{~nm}$ in our particular case). Observations of bright unpolarised standard stars indicate that any ripple generated by this retarder is smaller than $0.05 \%$ peak-to-peak, i.e. more than 20 times weaker than those induced by Halle retarder. Instead of using a half-wave plate, we rotate the polarimeter as a whole (by angles of $0^{\circ}, 45^{\circ}, 90^{\circ}$ and $135^{\circ}$ ); although slightly slower, less convenient (as automatic guiding needs to be retuned after each instrument rotation) and less accurate (see Sect. 3.1), this procedure turns out to be more achromatic than the original solution involving the Halle wave plate. Note that circular spectropolarimetry is only accurate within $400-700 \mathrm{~nm}$ in this temporary solution, and therefore requires a special "intermediate" setup of the MuSiCoS spectrograph between the two nominal "blue" and "red" configurations (Baudrand \& Böhm 1992). In this new setup, we can cover for instance the whole $450-$ $660 \mathrm{~nm}$ range in a single exposure.

\subsection{Beamsplitter}

Once the stellar polarisation of interest is converted (by means of retarders or polarimeter rotations) into a reference linear polarisation (with an electric field oriented along the reference azimuth defined in Sect. 2.2), we use

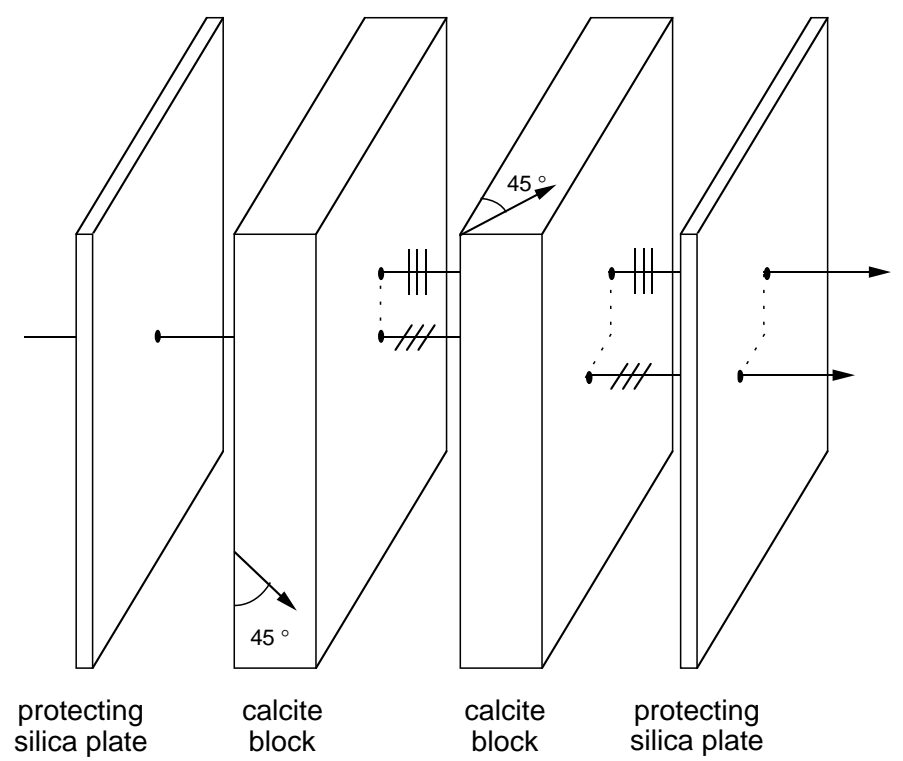

Fig. 3. Beamsplitter of the MuSiCoS polarimeter. The arrows depict the direction of the optical axes in each calcite block, while the hatch marks represent the direction of the electric field vibration of both ordinary and extraordinary rays. The two crystals and plates are glued together with an epoxy cement

a combination of birefringent crystals to split the stellar light into two beams corresponding respectively to polarisation along and perpendicular to the reference azimuth.

This beamsplitter (see Fig. 3) is made of two crossed calcite blocks (Savart plate). The advantage of such a design with respect to a conventional single block is that both beams emerging from the analyser are shifted by the same amount with respect to the incident beam. In particular, the chromatic variation of this splitting equally affects both beams, whose symmetry is therefore enhanced compared to the single block solution. The thickness of each calcite block $(3.84 \mathrm{~mm})$ ensures that both emerging beams are separated by $0.60 \pm 0.03 \mathrm{~mm}$ and shifted by $0.42 \pm 0.02 \mathrm{~mm}$ each with respect to the incident beam, throughout the whole spectral domain (390 to $870 \mathrm{~nm})$. This shift corresponds to the minimum possible separation between the two fibres in the image plane.

Note that a Savart plate is not free of astigmatism (e.g. Semel 1987). However, the large focal reduction the beam is subject to after the polarisation analysis (see Sect. 2.5) ensures that both tangential and sagittal images always fall within $3.2 \mu \mathrm{m}$ of the double fibre focal plane throughout the whole spectral range, implying that astigmatism can be easily neglected.

Our Savart plate was custom-built by Optique Jean Fichou in France. 


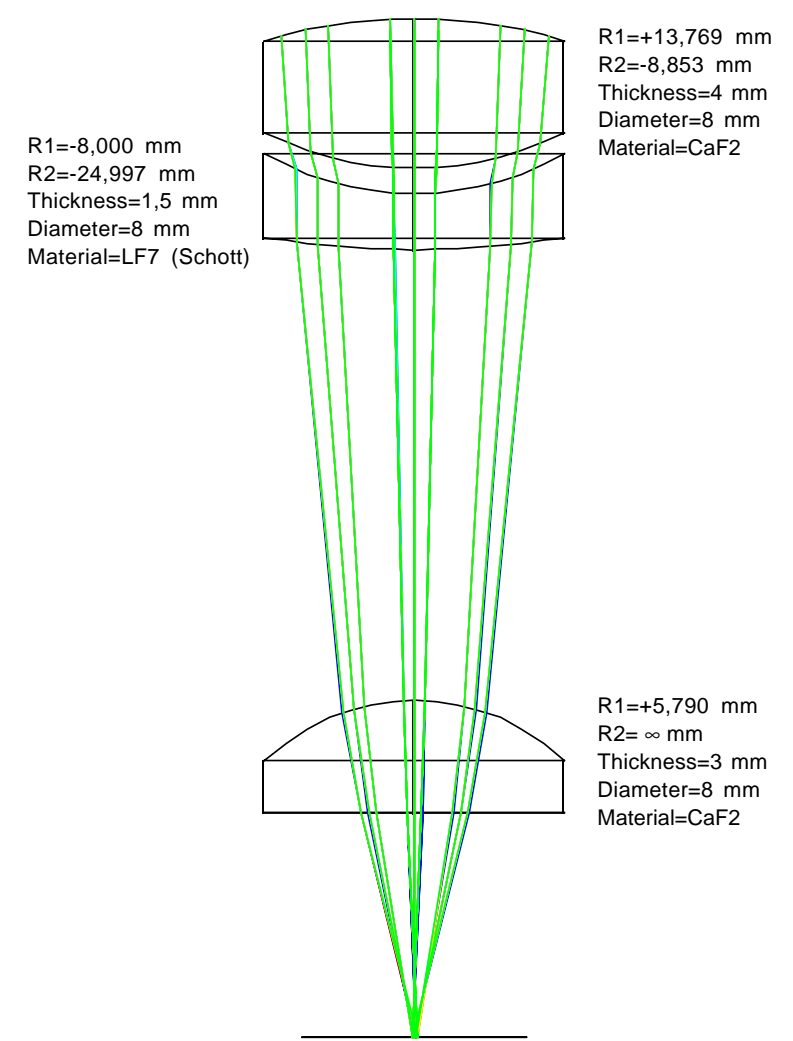

Fig. 4. Focal reducer of the MuSiCoS polarimeter. The Cassegrain beam comes from the top and the horizontal line at the bottom depicts the focal plane of the double fibre. $R 1$ and $R 2$ denote the curvature radii of the upper and lower surface of each lens respectively

\subsection{Focal reducer}

We then need a focal reducer to speed the beam up to an aperture of $\mathrm{f} / 2.5$, at which fibres behave satisfactorily.

Given the large desired magnification factor and the space into which all of the above optical components and mountings must be placed (see Fig. 1), a dedicated focal reducer had to be specially designed to minimise longitudinal chromatic aberrations. The selected design (see Fig. 4) includes three single lenses and ensures that the multicolour spot diagram is smaller than $5 \mu \mathrm{m}$ up to an object off-axis distance of $670 \mu \mathrm{m}$ (corresponding to the beamsplitter shift increased by the radius of the entrance aperture at a beam speed of $\mathrm{f} / 25$ ), only slightly larger than the diffraction limit.

This focal reducer and its mounting were built and assembled by Soptel Technologies in France.

\subsection{Optical fibres}

Two optical fibres with core/cladding diameters of $50 / 60 \mu \mathrm{m}$ (mounted side by side in a special connector)

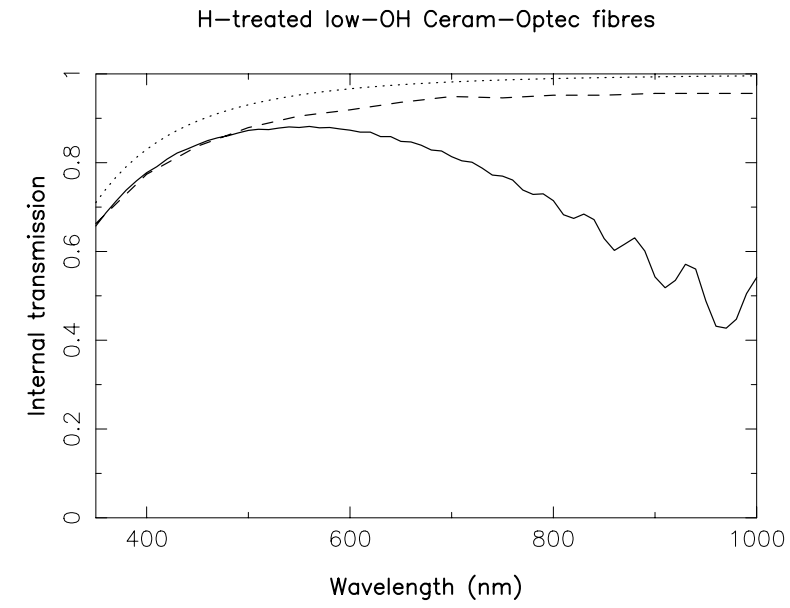

Fig. 5. Internal transmission of H-treated low-OH Ceram-Optec Optran fibres for 50/60 $\mu \mathrm{m}$ (full line) and 200/220 $\mu \mathrm{m}$ (dashed line) core/cladding diameters. The dotted line depicts the theoretical Rayleigh dispersion limit. All these curves correspond to $25 \mathrm{~m}$ fibres

finally collect the two orthogonally polarised beams and send them to the MuSiCoS spectrograph.

The optical fibre we selected is the prototype H-treated low-OH Ceram-Optec Optran fibre whose transmission is optimised in the $350-1100 \mathrm{~nm}$ wavelength band. Measurements at the European Southern Observatory (Garching laboratories) and Observatoire de Meudon have confirmed that the transmission of such fibres is very close to the Rayleigh limit all the way from 350 to $1000 \mathrm{~nm}$ for fibre core/cladding diameters of $200 / 220 \mu \mathrm{m}$ (see Fig. 5). The $50 / 60 \mu \mathrm{m}$ fibre we purchased for our purpose is just as good up to $500 \mathrm{~nm}$, but gets progressively worse at red and infrared wavelengths with an internal transmission as low as $50 \%$ at $1 \mu \mathrm{m}$ (for a $25 \mathrm{~m}$ fibre). According to CeramOptec engineers, this problem is very likely due to small fibre stresses induced by the acrylate jacket. Despite all efforts to date (in particular, attempting to employ a more flexible epoxy jacket), Ceram-Optec has not yet succeeded in producing a better fibre with the same core/cladding diameters. In the meantime, we have been using this preliminary fibre whose transmission turns out to be reasonably good in average in the restricted wavelength domain imposed by the Fichou quarter-wave plate $(400-700 \mathrm{~nm}$, see Sect. 2.3).

\subsection{Mechanical and electronic design}

The mechanical structure of the polarimeter has been made as rigid as possible to ensure that the entrance hole and double fibre remain optically aligned for all positions of the polarimeter and the telescope. This is indeed an important constraint for the overall instrument transmission, as any relative shift between the entrance aperture and fibre can generate a significant light loss. This loss reaches for instance $13 \%$ for a relative aperture/fibre shift 
of $10 \%$ of the aperture/fibre size (i.e. $50 \mu \mathrm{m}$ at the TBL entrance aperture or $5 \mu \mathrm{m}$ at fibre level). Therefore, the two fast beam portions of the instrument (entrance aperture/attendant doublet and focal reducer/fibre) have each been designed as two separate tight compact blocks. Both are fixed onto a larger parallelepiped structure (on the upper and lower sides, respectively) which supports the two rotatable wheels (holding the polarisers and retarders) and the beamsplitter. This structure is rigid enough to ensure that differential deformation between the upper and lower sides remains smaller than $10 \mu \mathrm{m}$ in all directions and for all telescope positions.

Two stepper motors (mounted on the main structure) are used to rotate the two wheels, while a third one (fixed on the wave plate wheel) rotates (through a gear chain) the two retarders simultaneously. Each motor is associated with a proximity detector defining a reference azimuth for the motor axis. Several solutions are usually available to check that no step is skipped during the rotation. The most obvious one, which consists in using potentiometers to measure the angular position of each rotatable unit, could not be applied here for lack of space. We decided instead to drill several notches in the circular edge of each of the four rotatable units (two wheels and two plate holders), one for each position at which each unit can be positioned (i.e. three for the polariser wheel, three for the retarder wheel, three for the quarter-wave plate holder and four for the half-wave plate holder); a small wheel (maintained with a spring onto the circular edge of each rotating unit) then indicates (through a microswitch) whether it is embedded into a notch, i.e. whether the unit has reached the correct angular position. Note that, in this second solution, the fine angular unit positioning is actually obtained through the wheel/notch interactions (if we allow enough gear backlash) rather than with the stepper motors themselves (only used here to drive units to an approximate azimuth).

The three stepper motors (purchased from the Swiss company ESCAP) are remote controlled through a commercial MCU11-STD microcontroller card (built by the French company EPILOG and including a 68HC11A1 microcontroller, a $32 \mathrm{~kb}$ EEPROM loaded with dedicated software developed at Observatoire Midi-Pyrénées, a $24 \mathrm{~kb}$ RAM and an input/output port controller) as well as an interface card (developed at Observatoire MidiPyrénées and involving in particular L297 and L298N SGS-Thomson clock and power drivers). Thirteen twocharacter commands (sent from a terminal to the microcontroller through a serial port) enable one to obtain all possible instrument configurations ( $\mathrm{p} 1$ and $\mathrm{p} 3$ for the circular and linear polarisers respectively, $\mathrm{p} 2$ for no polariser; 11 and 13 for the quarter and half-wave plates respectively, 12 for no wave plate; q1, q2 and q3 for quarterwave plate azimuths $-45^{\circ}, 0^{\circ}$ and $45^{\circ}$ respectively; d1, $\mathrm{d} 2, \mathrm{~d} 3$ and $\mathrm{d} 4$ for half-wave plate azimuths $0^{\circ}, 22.5^{\circ}, 45^{\circ}$ and $67.5^{\circ}$ respectively). For each command, the corre- sponding stepper motor first rotates back to its axis reference azimuth (see previous paragraph), switches direction and does a preset number of steps. Once the stepper motor has stopped, the microcontroller then automatically checks (through the corresponding microswitch) whether the instrument has safely reached the requested position. An error message is sent to the user if any problem is encountered (e.g. wrong command, no microswitch contact).

\subsection{Tuning procedure}

Tuning the instrument consists in aligning the axes of the various birefringent optical components, then perfectly aligning the entrance aperture of the polarimeter with the two optical fibres. This is achieved with the four following steps.

We first mount the instrument on an optical bench, send light through the instrument aperture, remove the fibre and look at the double image with a microscope. We then set the linear polariser of the polarimeter inside the beam leaving the two retarders out (p3 12 configuration). We then manually rotate the beamsplitter to achieve the best possible extinction of one beam, i.e. to align its optical axes with those of the linear polariser.

In a second step, we insert the half-wave plate in the beam and set its holder to an azimuth of $0^{\circ}$ (13 d1 configuration). We then rotate the plate manually within its holder to restore the beam extinction obtained in the previous step. We repeat the same operation with the quarterwave plate in the beam, with its holder set to an azimuth of $0^{\circ}$ (11 q2 configuration).

In a third step, we mount the double fibre back in position onto the polarimeter and send light through it from its other end. Both polarisers and retarders are removed from the beam (p2 12 configuration). We then dismount the entrance aperture/attendant doublet block from the main polarimeter structure and observe with the microscope the image of the double fibre duplicated by the beamsplitter. Superimposing two of the four images by rotating the fibre/focal reducer block with respect to the main polarimeter structure ensures that the two fibres are aligned onto the analyser duplication direction.

The last step consists in adjusting very precisely the entrance aperture/attendant doublet block onto the two overlapping central images of the double fibre, in both lateral and longitudinal directions. In a final check, we verify that inserting the polarisers and retarders into the beam or rotating the wave plates generate no apparent beam deviation.

\section{First results}

Operating the MuSiCoS polarimeter is very simple. Once the correct instrument configuration has been selected 
Efficiency of MuSiCoS spectropolarimeter

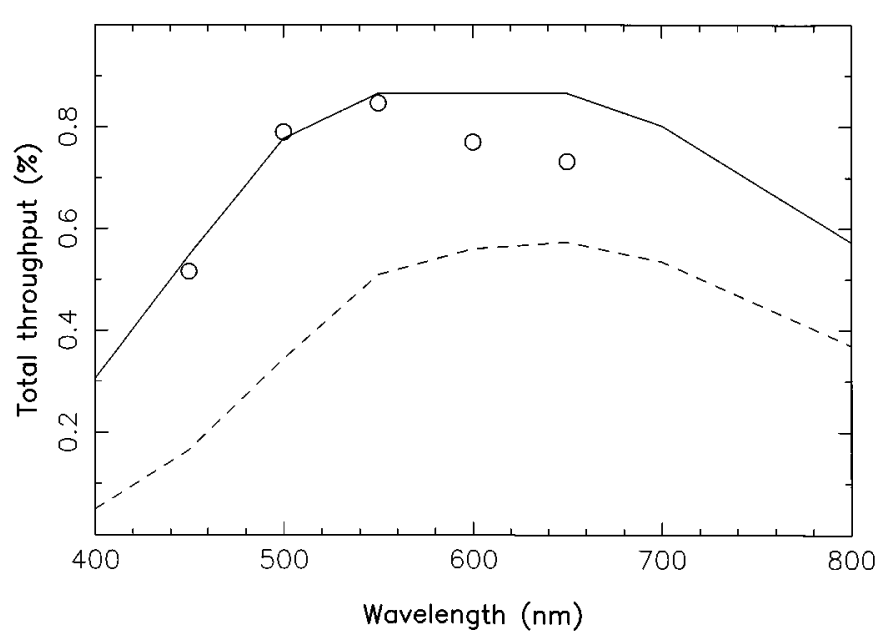

Fig. 6. Total efficiency of MuSiCoS spectropolarimeter at TBL (counted from above the atmosphere down to the detector, detector included). The solid line depicts the nominal spectrograph efficiency with the new SITE detector and Ceram-Optec optical fibre scaled from the original values (dashed line) of Baudrand \& Böhm (1992). Open symbols correspond to the best measurements obtained in the spectropolarimetric setup

(e.g. p2 11 for circular polarisation), we usually run sequences of four subexposures on the stellar object of interest with alternating wave plate or instrument azimuth (e.g. q1, q3, q3 and q1 for Stokes $V$ measurements). Data reduction and polarisation information extraction is then achieved with a dedicated code called ESpRIT, the detailed description of which is given in Donati et al. (1997). This package is installed on the DecAlpha workstation in the TBL control room, where it takes typically $5 \mathrm{~min}$ to process a sequence of four polarisation exposures.

This instrument has been used for four runs already, three times at TBL (August 1996, February 1997 and February 1998) and once in Hawaii (November 1996) within the framework of the MuSiCoS '96 international campaign. It is now fully tested and available most of the time at TBL (between the MuSiCoS campaigns in which it is involved) to the entire astronomical community.

The global efficiency is found to be similar to that of the $\mathrm{MuSiCoS}$ spectrograph used in non-polarimetric mode (see Baudrand \& Böhm 1992) when we take into account the increased efficiency of the new SITE CCD detector (now available at TBL) and the fibre transmission problem mentioned in Sect. 2.6 (see Fig. 6). Altogether, we obtain $\mathrm{S} / \mathrm{N}$ of $275,345,355,335$ and 320 per $4.4 \mathrm{~km} \mathrm{~s}^{-1}$ pixel on an A0 star with $m_{V}=7.0$ in a $1 \mathrm{hr}$ exposure at wavelengths of 450, 500,550, 600 and $650 \mathrm{~nm}$ respectively.
LSD profiles of the Sun
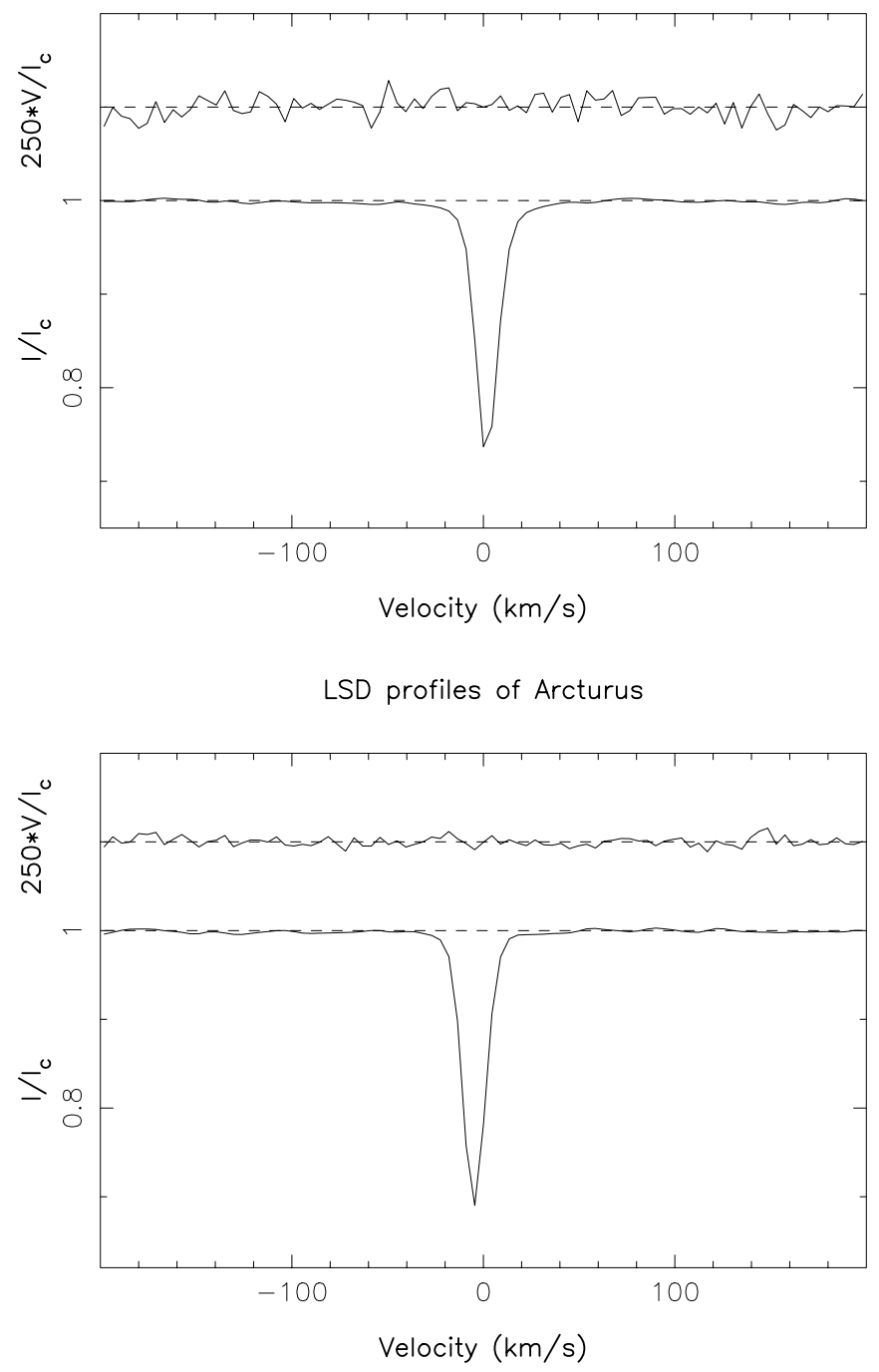

Fig. 7. LSD unpolarised (bottom curve of each panel) and circularly polarised (top curve of each panel) profiles, for the Sun (top panel) and Arcturus (bottom panel). Note that the LSD Stokes $V$ spectrum are shifted upwards by 1.1 and expanded 250 times in these plots

\subsection{Polarisation in line profiles}

We find that the instrument performs very well for detecting polarisation in line profiles.

We first checked that no polarisation signature are detected in line profiles of slowly rotating, weakly active standard stars like the Sun or Arcturus, whose disc integrated magnetic field and associated Zeeman signatures are extremely weak. With the new LSD crosscorrelation technique (Donati et al. 1997), one can extract a "mean polarisation signature" (called LSD profile) from all recorded spectral lines simultaneously, and increase considerably the accuracy to which such polarisation signals can be detected. With such a technique, we can check for instance that no Stokes $V$ signatures are 
detected on the Sun or on Arcturus down to a relative rms noise level of $0.004 \%$ and $0.002 \%$ respectively (see Fig. 7). We can conclude in particular that no spurious circular polarisation signals (due to stellar rotation and variability, Earth rotation, drifts in the spectrograph, inhomogeneities in CCD pixel sensitivities, see Donati et al. 1997, for a complete discussion) are observed for these two objects, and that our instrument, observing procedure and processing software behave correctly.

Very weak signals (with a peak-to-peak amplitude of a few $0.01 \%$ ) are however detected in two LSD linear polarisation profiles of Arcturus, suggesting that the process of rotating the instrument between successive subexposures (see Sect. 2.3) probably induces systematic low level velocity shifts in the corresponding spectra (at a level of 10 to $20 \mathrm{~m} \mathrm{~s}^{-1}$ typically) and therefore spurious polarisation signatures such as those discussed in Donati et al. (1997). Their amplitude is however sufficiently small (much smaller in particular than any of the true linear polarisation profiles we detected for the magnetic programme stars as shown below, and very often below the noise level itself) that we decided to keep using the same observing procedure (involving instrument rotations), at least till the Halle wave plate problem is fixed (see Sect. 2.3).

The first main scientific goal of our instrument is the study of magnetic field topologies of rapidly rotating active stars through the Zeeman signatures they generate in the spectral line profiles of these objects. Figure 8 presents LSD Stokes $V$ profiles of two of the most active RS CVn systems (HR 1099 and II Peg) obtained from MuSiCoS spectropolarimetric data recorded in 1998 February. It is obvious from these plots that Stokes $V$ Zeeman signatures are clearly detected on both stars (with relative amplitudes of $0.12 \%$ and $0.54 \%$, i.e. at an accuracy level of $11 \sigma$ and $31 \sigma$, respectively) and are very similar in size and shape to those measured already on these stars with other instruments (Donati et al. 1992a, 1997). Note in particular that the LSD Stokes $V$ signature of II Peg presented in Fig. 8 is the strongest ever detected on any cool stars to date. Monitoring such Zeeman signatures throughout a full stellar rotational cycle allows one to map the parent surface magnetic field topology using techniques of indirect stellar surface imaging (Doppler imaging). This new method (called Zeeman-Doppler imaging or ZDI) is found to be successful at recovering both location and shape of stellar magnetic regions as well as field strength and orientation within these regions (Donati \& Brown 1997). It therefore provides a new and original way of investigating stellar dynamos in general (Donati et al. 1992b, 1998; Donati \& Cameron 1997; Donati 1998). Such sets of rotationally modulated LSD Stokes $V$ profiles have already been collected with this instrument for 4 RS CVn systems to date, the detailed analysis of which will be published in a forthcoming paper. Linear polarisation Zeeman signatures are in principle also present in spectral lines of cool active stars but are likely too weak (typically ten times
LSD profiles of HR 1099

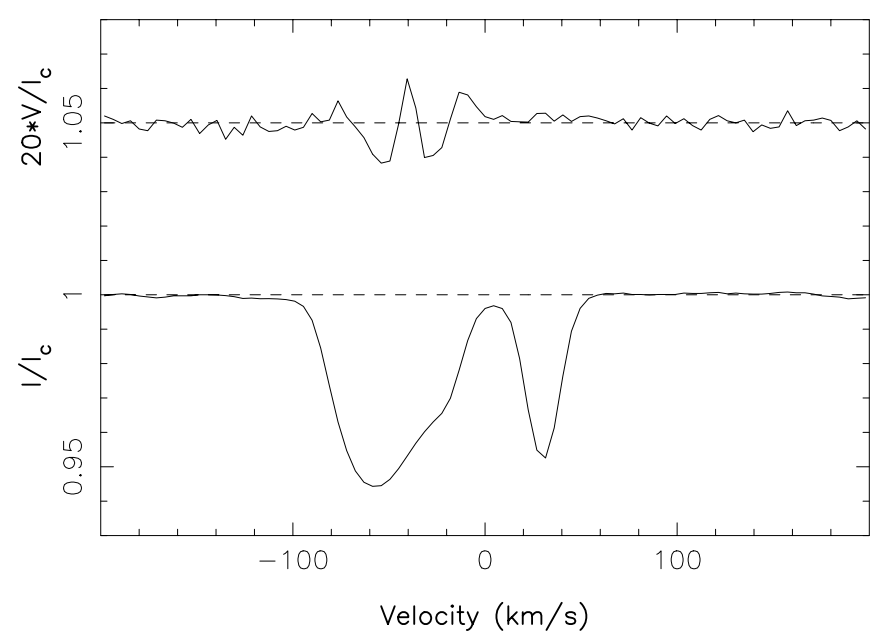

LSD profiles of II Peg

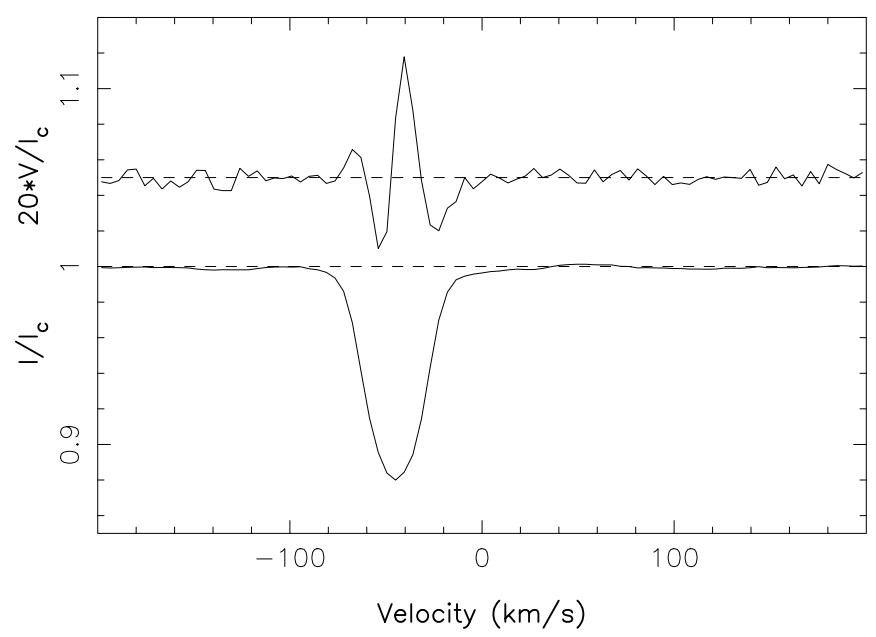

Fig. 8. LSD unpolarised (bottom curve of each panel) and circularly polarised (top curve of each panel) profiles of the rapidly rotating very active RS CVn systems HR 1099 (top panel) and II Peg (bottom panel), recorded on 1998 Feb. 05 and Feb. 16 respectively. The line profiles of the two system components are clearly visible in the unpolarised LSD profile of the double-line spectroscopic binary HR 1099 (K1IV+G2V) while only one line profile is visible for the single-line binary II Peg (K2IV). Note that the LSD Stokes $V$ spectra are expanded 20 times and shifted upwards by 1.05 in these plot

weaker than their Stokes $V$ equivalent) to be detected with our instrument.

The MuSiCoS spectropolarimeter can also be used to study the details of the magnetic topologies of magnetic chemically peculiar stars using both circular and linear Zeeman signatures which magnetic fields induce in spectral line profiles. As opposed to field topologies of active stars (consisting essentially of multiple spot distributions), field geometries of magnetic Ap/Bp stars can be described to first order as low-order axisymmetric multipole 
expansions. However, significant departures from such simple models (in the form of localised regions with enhanced radial field on the magnetic equator) have been detected already in several objects (e.g. Leroy et al. 1995, 1996; Wade et al. 1996). In particular, studying the nature and spatial distribution of these distortions should bring us some new insight on the still debated origin of magnetic fields in $\mathrm{Ap} / \mathrm{Bp}$ stars. Unfortunately, full reconstruction of the surface magnetic topologies of such objects with no a priori assumption about their large scale structure is impossible from circular spectropolarimetry alone, as demonstrated by Brown et al. (1991). Although it is still unclear yet whether sets of rotationally modulated LSD Stokes $Q$ and $U$ profiles will provide the missing information for such an imaging task, it is nevertheless obvious already from Leroy et al.'s results $(1995,1996)$ that linear polarisation profiles in spectral lines of magnetic Ap/Bp stars will greatly improve our description of how the field topologies of these chemically peculiar stars depart from low-order axisymmetric multipole expansions.

Our instrument can successfully collect such information, as demonstrated in Fig. 9 in the particular case of the Ap star 78 Vir. The corresponding longitudinal field (estimated with the method of Donati et al. 1997) is equal to $800 \pm 50 \mathrm{G}$, in good agreement with the published estimates of Borra \& Landstreet (1980). Similarly, the integrated Stokes $Q$ and $U$ profiles we measure yield average linear polarisation angles and signs that are perfectly compatible with those determined by Leroy (1995) from broadband spectropolarimetry. It is also obvious from our observations that LSD Stokes $Q$ and $U$ Zeeman signatures are much more informative on the actual field structure than broadband measurements. For instance, the LSD Stokes $Q$ profile of 78 Vir at rotational phase 0.650 is obviously non zero, although its average value implies a very weak level of broadband Stokes $Q$ linear polarisation in agreement with the results of Leroy (1995). Similarly, $\mathrm{MuSiCoS}$ spectropolarimetric observations of 53 Cam at rotational phase 0.827 (i.e. when broadband measurements indicate a very low amount of linear polarisation, Leroy 1995) show that Stokes $Q$ and $U$ profiles, while null in average, possess a rather complex shape and should therefore be very helpful for constraining more accurately the modelling of many fine details of the surface magnetic field structure. Up to now, we have secured data sets with good to very good phase coverage for five well-known magnetic Ap stars in all Stokes parameters, whose interpretation is underway and will be published in a forthcoming paper.

Finally, one can note from Figs. 9 and 10 that linear polarisation profiles are much weaker than their circular polarisation equivalent, often 15 to 20 times smaller. It is therefore very important to check for potential circular to linear polarisation instrumental crosstalk; observations of Ap stars indicate that this crosstalk rate is lower than $0.5 \%$ of the Stokes $V$ amplitude for our polarimeter.
LSD profiles of $78 \mathrm{Vir}$

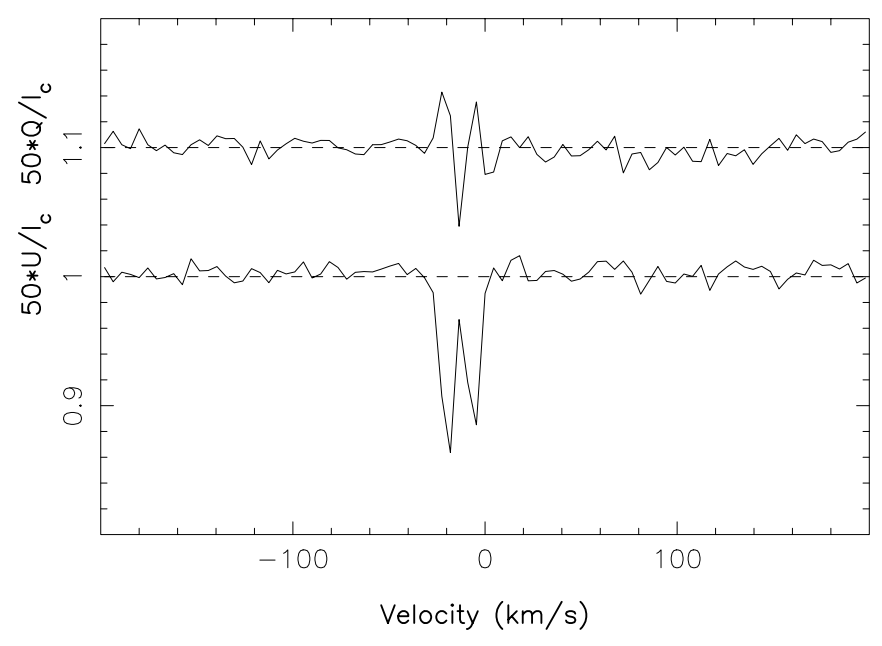

LSD profiles of $78 \mathrm{Vir}$

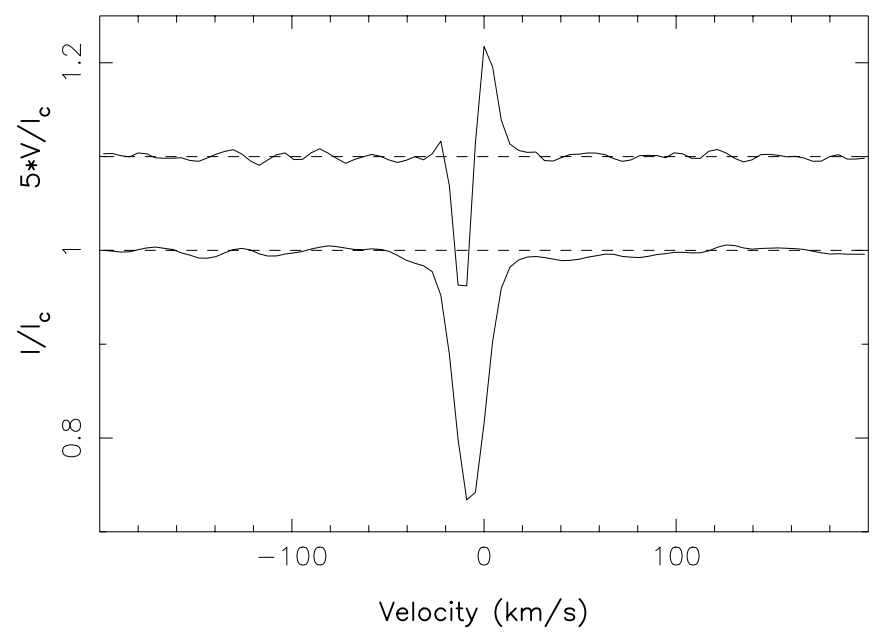

Fig. 9. LSD Stokes $Q, U, V$ and $I$ profiles (from top to bottom) of 78 Vir recorded on 1998 Feb. 18 at rotational phase 0.650 (using ephemeris JD $=2434816.90+3.7220 E$ ). Note that Stokes $Q$ and $U$ profiles were expanded by 50 and shifted upwards by 1.1 and 1.0 respectively, while the Stokes $V$ spectrum is expanded by 5 and shifted upwards by 1.1 on these plots

Other scientific applications (e.g. search for flare induced linear polarisation signatures in Balmer lines of active stars, Saar et al. 1994) should in principle be accessible to our instrument, but have not been attempted yet.

\subsection{Continuum polarisation and line depolarisation structures}

The MuSiCoS spectropolarimeter can in principle also estimate continuum polarisation in stellar spectra. This observing mode relies on measuring relative continuum flux variations between the two orthogonally polarised beams that correlate with wave-plate rotations. The main problem here consists in reducing true and spurious 
LSD profiles of $53 \mathrm{Cam}$

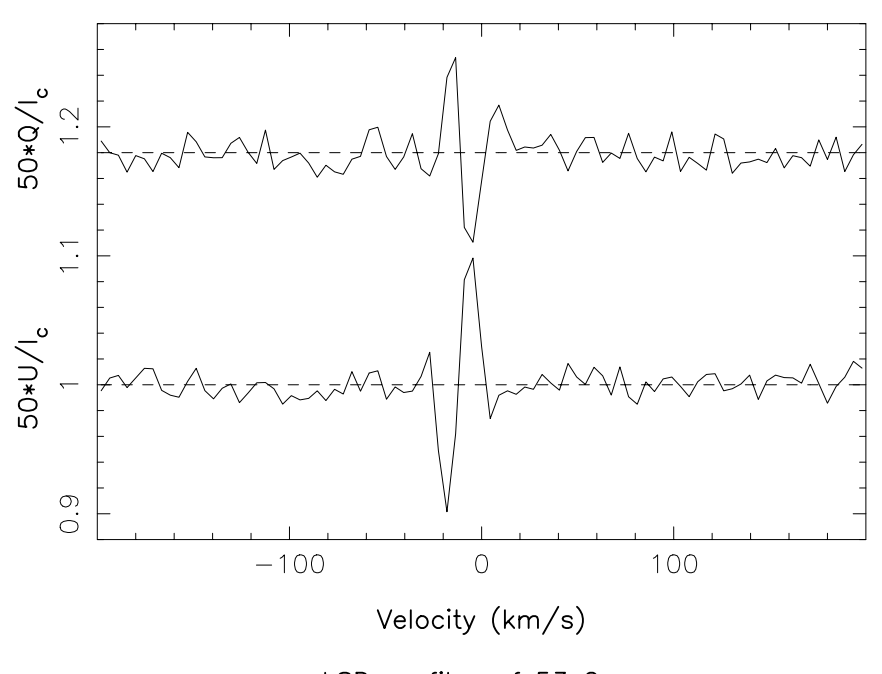

LSD profiles of $53 \mathrm{Cam}$

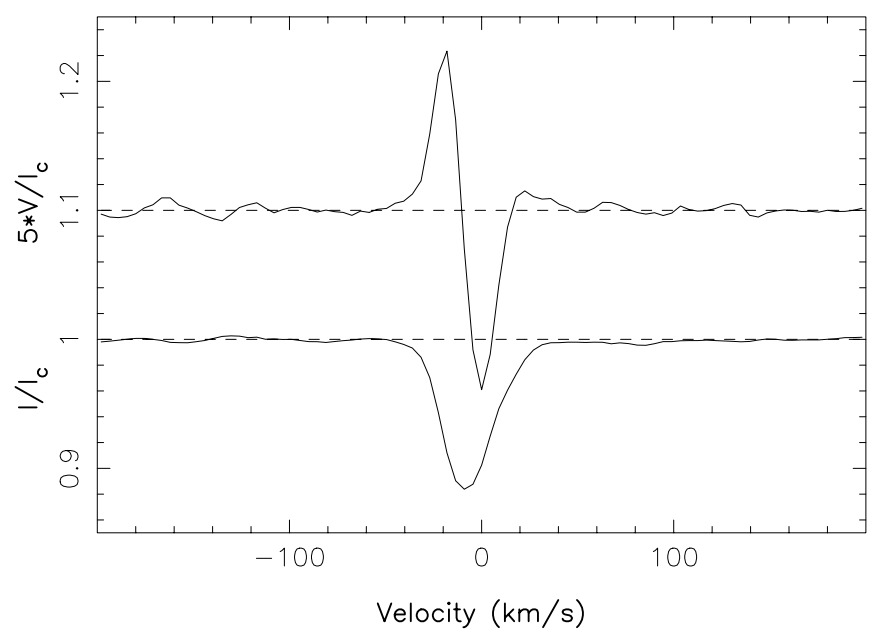

Fig. 10. LSD Stokes $Q, U, V$ and $I$ profiles (from top to bottom) of 53 Cam recorded on 1997 Feb. 25 at rotational phase 0.827 (using the new ephemeris JD $=2448500.193+8.02681 E$ of Hill et al. 1998, with phase 0.0 referring to the positive maximum in longitudinal field). Note that Stokes $Q$ and $U$ profiles were expanded by 50 and shifted upwards by 1.18 and 1.0 respectively, while the Stokes $V$ spectrum is expanded by 5 and shifted upwards by 1.1 on these plots

instrumental polarisation to a minimum. If the true instrumental polarisation (generated through the successive reflections onto the primary and secondary mirrors) is usually very small at the Cassegrain focus (of the order of $0.01 \%$ at TBL as estimated with the Sterenn photopolarimeter), spurious instrumental polarisation can be potentially very important. Such spurious signatures may indeed come from potential motions of the double image at fibre level (induced by wave plate rotations, instrument flexures with telescope movement or simply small random fluctuations in light injection) coupled to slight differential mispositioning of the two images onto the two fibres (due to small magnification errors in the focal reducer, to slight azimuthal misalignment of the analyser with respect to the fibre or to the chromatism of the beamsplitter).

This spurious polarisation is expected to depend only weakly on wavelength and is virtually undistinguishable from true continuum polarisation; while it is not a problem when measuring polarisation in line profiles (as we usually remove a posteriori both true and spurious continuum polarisation from the observations in this case), it can be very damaging when one is interested in studying the continuum polarisation itself, from scattering circumstellar environments for instance. As we estimate that potential mispositioning of each image onto its corresponding fibre is typically be of the order of 1 to $2 \mu \mathrm{m}$, we expect possible variations in the continuum flux emerging each fibre of about $2 \%$ rms and spurious polarisation levels from a sequence of four subexposures (i.e. eight spectra) of $\pm 0.7 \%$ (if the image mispositioning and associated spurious continuum flux variation does not correlate with the azimuth of the wave plate or instrument) or larger (otherwise). In practice, observations of unpolarised standard stars indicate that spurious continuum polarisation is of the order of $0.8 \% \mathrm{rms}$ in circular polarimetry, in good agreement with the theoretical lower limit derived above. It implies in particular that wave plate rotations generate only very weak systematic image displacement at fibre input. In linear polarimetry however, the level of spurious continuum polarisation is about twice larger, indicating that the instrument rotations required in our observing procedure do produce systematic image motions at fibre input and therefore larger spurious continuum polarisation. Similarly, the accuracy to which gradients in continuum polarisation (between both edges of our spectral domain) can be estimated is typically $0.5 \%$ rms for Stokes $V$ observations, and about twice as much for Stokes $Q$ and $U$ observations.

Altogether, the MuSiCoS spectropolarimeter is therefore poorly competitive at measuring stellar continuum polarisation with respect to photopolarimeters like Sterenn or Cassegrain low-resolution spectropolarimeters, which are typically two orders of magnitude more accurate for that particular purpose (e.g. Leroy 1995). This is unfortunately an intrinsic limitation of our $50 \mu \mathrm{m}$ fibre setup.

The MuSiCoS spectropolarimeter should nevertheless be very useful for studying emission lines (and in particular, forbidden lines) depolarisation structures with respect to the surrounding continuum, and thus for determining where these lines form with respect to the scattering environment. Such depolarisation structures have for instance been recently observed in the case of the $\mathrm{O}$ star $\theta^{1}$ Ori $\mathrm{C}$ (see Fig. 11), at an epoch where the continuum level was circularly polarised at a rate of a few $\%$. The fact that the emission lines of nebular origin (the [N II] lines of multiplet $1 \mathrm{~F}$ at 654.81 and $658.36 \mathrm{~nm}$, the central peak of $\mathrm{H} \alpha$, but also the [O III] lines of multiplet $1 \mathrm{~F}$ at 495.89 
$\mathrm{H} \alpha$ Stokes $V$ profile of $\theta^{1}$ Ori $C$

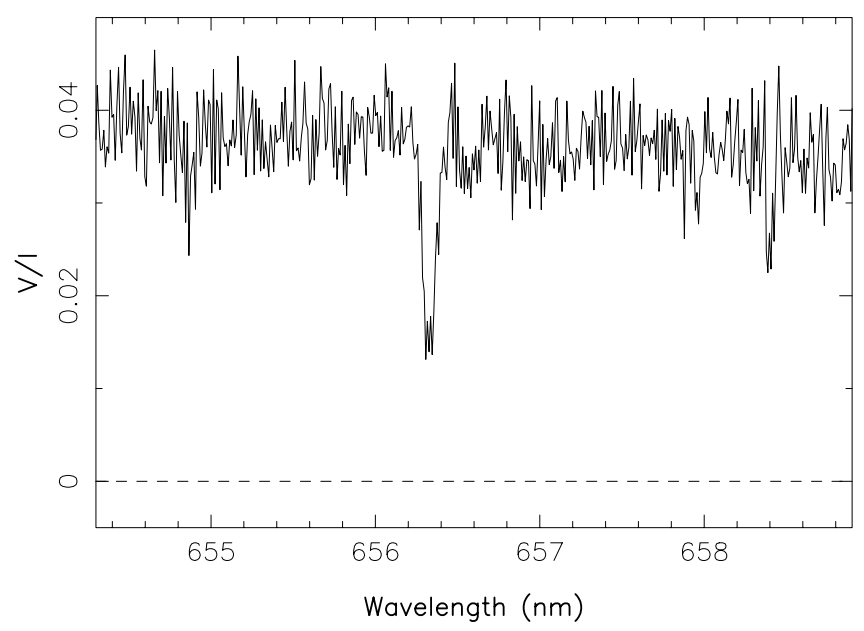

$\mathrm{H} \alpha$ Stokes I profile of $\theta^{1}$ Ori $\mathrm{C}$

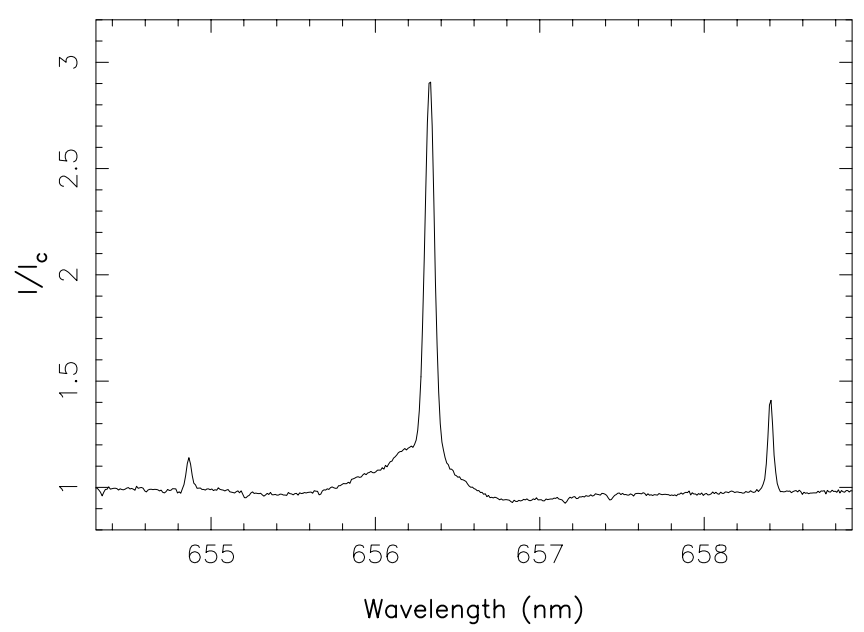

Fig. 11. Circular polarisation rate (top panel) and normalised intensity (bottom panel) of $\theta^{1}$ Ori $\mathrm{C}$ at $\mathrm{H} \alpha$ wavelengths, on 1997 Feb. 20

and $500.68 \mathrm{~nm}$ ) are all depolarised in the same proportion as the line-to-continuum flux ratio indicates that all these lines are formed outside the scattering circumstellar environment and that the continuum circular polarisation is therefore of stellar (rather than interstellar) origin. A more extensive description of these results is presented in another paper (Donati \& Wade 1998).

Another potential (but yet untested) scientific application of our instrument in this field is the study of how strong winds of $\mathrm{O}$ stars depart from spherical symmetry through the depolarisation of spectral lines formed in the wind (Lefèvre 1992; Harries \& Howarth 1996).

As continuum polarisation or line depolarisation structures produced by scattering circumstellar environments are usually an order of magnitude stronger in linear than in circular polarisation, one is usually concerned by the rate at which the instrument converts linear to circular polarisation (rather than the opposite as in Sect. 3.1). A circular analysis of $100 \%$ linearly polarised light (obtained by flat-field illumination through the linear sheet polariser located in the first polarimeter wheel, see Sect. 2.2) indicates that the crosstalk level from linear to circular polarisation is only $0.2 \%$.

\section{Conclusion}

We have presented in this paper the technical characteristics of a new polarimeter dedicated to the MuSiCoS échelle spectrograph that can collect high resolution circularly and linearly polarised spectra of stellar objects.

This polarimeter was initially designed to be very achromatic in the 390 to $870 \mathrm{~nm}$ range, by using in particular the Halle superachromatic crystalline retarders. However, we discovered that such wave plates generate large amplitude ( $1-2 \%$ peak-to-peak) intensity and polarisation ripples and turn out to be unusable for our purpose. We replaced the quarter-wave retarder with a less achromatic Fichou plate (for which such ripples are at least 20 times smaller), at the expense of restricting circular spectropolarimetric experiments to a 400 to $700 \mathrm{~nm}$ interval. We avoid using a half wave plate by simply rotating our instrument at specific azimuths.

We find that this instrument is very good at measuring polarisation or depolarisation structures in line profiles, with a relative accuracy of down to at least $0.002 \%$. It is therefore one of the only facilities worldwide for studying magnetic topologies of active and chemically peculiar stars (through rotational modulation of linearly and circularly polarised Zeeman signatures in line profiles). It should also be a very interesting tool for investigating geometries of non-axisymmetric circumstellar environments (through depolarisation of spectral lines formed within the scattering envelope). This instrument could in principle also be used to estimate linear and circular continuum polarisation of stellar objects, but our fibre setup limits the accuracy of such measurements to a level of about $1 \%$.

Additional up to date information on the $\mathrm{MuSiCoS}$ spectropolarimeter (in its TBL configuration) and on its operation is available on the world-wide-web at URL http://www.obs-mip.fr/omp/umr5572/magnetisme/polarmus.html.

Acknowledgements. We thank Cyril Delaigue for writing the dedicated microcontroller code, Laurent Parès for a few additional ray tracing computations, Gerardo Avila and Jacques Baudrand for measuring the transmission of our optical fibre, Torsten Böhm and John Landstreet for their involvement in data collection, and Meir Semel, François Ménard, Jean-Louis Leroy and John Landstreet for discussions on polarisation and polarimetric devices. We are also grateful to the referee, Ian Howarth, for his careful reading of the manuscript. 


\section{References}

Baudrand J., Böhm T., 1992, A\&A 259, 711

Borra E.F., Landstreet J.D., 1980, ApJS 42, 421

Brown S.F., Donati J.-F., Rees D.E., Semel M., 1991, A\&A 250, 463

Catala C., Foing B.H., Baudrand J., et al., 1993, A\&A 275, 245

Chrysostomou A., Ménard F., Gledhill T.M., et al., 1997, MNRAS 285, 750

Donati J.-F., 1998, MNRAS (in press)

Donati J.-F., Brown S.F., 1997, A\&A 326, 1135

Donati J.-F., Brown S.F., Semel M., et al., 1992b, A\&A 265, 682

Donati J.-F., Cameron A.C., 1997, MNRAS 291, 1

Donati J.-F., Cameron A.C., Hussain G.A.J., Semel M., 1998, MNRAS (in press)

Donati J.-F., Semel M., Carter B.D., Rees D.E., Cameron A.C., 1997, MNRAS 291, 658

Donati J.-F., Semel M., Rees D.E., 1992a, A\&A 265, 669
Donati J.-F., Wade G.A., 1998, A\&A (in press)

Harries T.J., Howarth I.D., 1996, A\&A 310, 533

Hill G.M., Bohlender D.A., Landstreet J.D., et al., 1998, MNRAS 297, 23

Lefèvre J., 1992, A\&A 254, 274

Leroy J.-L., 1995, A\&AS 114, 79

Leroy J.-L., Landolfi M., Landi Degl'Innocenti E., 1996, A\&A 311,513

Leroy J.-L., Landolfi M., Landi Degl'Innocenti M., et al., 1995, A\&A 301, 797

Saar S.H., Martens P.C.H., Huovelin J., Linnaluoto S., 1994, A\&A 286, 194

Semel M., 1987, A\&A 178, 257

Semel M., Donati J.-F., Rees D.E., 1993, A\&A 278, 231

Serkowski K., 1974, in: Carleton N.P. (ed.), "Methods of Experimental Physics", Vol. 12, Part A. Academic, New York, p. 361

Wade G.A., Elkin V.G., Landstreet J.D., Leroy J.-L., Mathys G., Romanyuk I.I., 1996, A\&A 313, 209 